This is an electronic reprint of the original article. This reprint may differ from the original in pagination and typographic detail.

Author(s): Tarvainen, Olli; Kalvas, Taneli; Koivisto, Hannu; Komppula, Jani; Kronholm, Risto; Laulainen, Janne; Izotov, I.; Mansfeld, D.; Skalyga, V.; Toivanen, V.; Machicoane, G.

Title: Limitation of the ECRIS performance by kinetic plasma instabilities (invited)

Year: $\quad 2016$

Version:

Please cite the original version:

Tarvainen, O., Kalvas, T., Koivisto, H., Komppula, J., Kronholm, R., Laulainen, J., Izotov, I., Mansfeld, D., Skalyga, V., Toivanen, V., \& Machicoane, G. (2016). Limitation of the ECRIS performance by kinetic plasma instabilities (invited). Review of Scientific Instruments, 87(2), Article 02A703. https://doi.org/10.1063/1.4931716

All material supplied via JYX is protected by copyright and other intellectual property rights, and duplication or sale of all or part of any of the repository collections is not permitted, except that material may be duplicated by you for your research use or educational purposes in electronic or print form. You must obtain permission for any other use. Electronic or print copies may not be offered, whether for sale or otherwise to anyone who is not an authorised user. 


\section{A|P|P $\mid \begin{aligned} & \text { Review of } \\ & \text { Scientific Instruments }\end{aligned}$}

\section{Limitation of the ECRIS performance by kinetic plasma instabilities (invited)}

O. Tarvainen, T. Kalvas, H. Koivisto, J. Komppula, R. Kronholm, J. Laulainen, I. Izotov, D. Mansfeld, V.

Skalyga, V. Toivanen, and G. Machicoane

Citation: Review of Scientific Instruments 87, $02 A 703$ (2016); doi: 10.1063/1.4931716

View online: $\mathrm{http}: / / d x$. doi.org/10.1063/1.4931716

View Table of Contents: http://scitation.aip.org/content/aip/journal/rsi/87/2?ver=pdfcov

Published by the AIP Publishing

\section{Articles you may be interested in}

A Hamiltonian fluid-kinetic model for a two-species non-neutral plasma

Phys. Plasmas 21, 044504 (2014); 10.1063/1.4871491

Nonaxisymmetric magnetorotational instability in ideal and viscous plasmas

Phys. Plasmas 15, 052103 (2008); 10.1063/1.2907788

Effective plasma confinement by applying multipolar magnetic fields in an internal linear inductively coupled plasma system

Appl. Phys. Lett. 88, 161503 (2006); 10.1063/1.2188037

Trapped electron effects in a magnetic filter field of the plasma source

Phys. Plasmas 11, 1735 (2004); 10.1063/1.1669391

Simulations and theories of relativistic ion cyclotron instabilities driven by $\mathrm{MeV}$ alpha particles in thermal deuterium plasmas

Phys. Plasmas 10, 1315 (2003); 10.1063/1.1561611

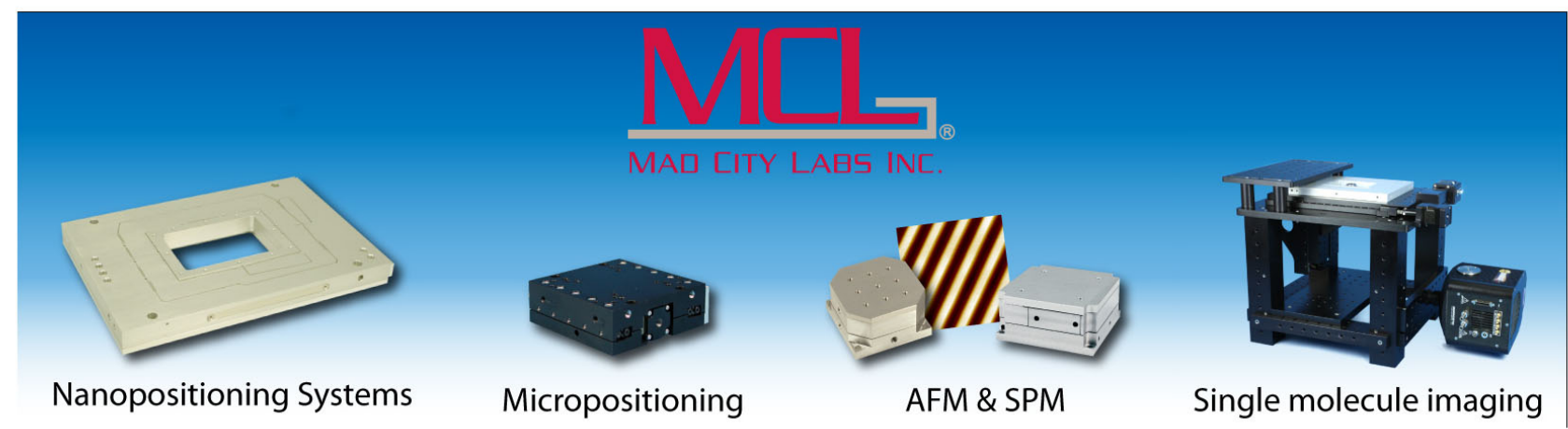




\title{
Limitation of the ECRIS performance by kinetic plasma instabilities (invited)
}

\author{
O. Tarvainen, ${ }^{1, a)}$ T. Kalvas, ${ }^{1}$ H. Koivisto, ${ }^{1}$ J. Komppula, ${ }^{1}$ R. Kronholm,${ }^{1}$ J. Laulainen, ${ }^{1}$ \\ I. Izotov, ${ }^{2}$ D. Mansfeld, ${ }^{2}$ V. Skalyga,,${ }^{2,3}$ V. Toivanen, ${ }^{4}$ and G. Machicoane ${ }^{5}$ \\ ${ }^{1}$ Department of Physics, University of Jyväskylä, 40500 Jyväskylä, Finland \\ ${ }^{2}$ Institute of Applied Physics, RAS, 46 Ul'yanova St., 603950 Nizhny Novgorod, Russian Federation \\ ${ }^{3}$ Lobachevsky State University of Nizhny Novgorod (UNN), 23 Gagarina St., \\ 603950 Nizhny Novgorod, Russian Federation \\ ${ }^{4}$ European Organization for Nuclear Research (CERN), 1211 Geneva 23, Switzerland \\ ${ }^{5}$ National Superconducting Cyclotron Laboratory, Michigan State University, \\ East Lansing, Michigan 48824, USA
}

(Presented 26 August 2015; received 21 August 2015; accepted 4 September 2015; published online 29 September 2015)

\begin{abstract}
Electron cyclotron resonance ion source (ECRIS) plasmas are prone to kinetic instabilities due to anisotropic electron velocity distribution. The instabilities are associated with strong microwave emission and periodic bursts of energetic electrons escaping the magnetic confinement. The instabilities explain the periodic ms-scale oscillation of the extracted beam current observed with several high performance ECRISs and restrict the parameter space available for the optimization of extracted beam currents of highly charged ions. Experiments with the JYFL $14 \mathrm{GHz}$ ECRIS have demonstrated that due to the instabilities the optimum $B_{\min }$-field is less than $0.8 B_{\mathrm{ECR}}$, which is the value suggested by the semiempirical scaling laws guiding the design of ECRISs. () 2015 AIP Publishing LLC. [http://dx.doi.org/10.1063/1.4931716]
\end{abstract}

\section{INTRODUCTION}

The magnetic field of a modern Electron Cyclotron Resonance Ion Source (ECRIS) is a superposition of solenoid and sextupole fields forming a so-called minimum-B topology. The magnetic field configuration serves for three purposes: (i) it provides a closed surface with constant magnetic flux density satisfying the condition for resonant energy transfer from the microwave radiation to the plasma electrons, i.e., $\omega_{R F}$ $=e B_{\mathrm{ECR}} / m_{e}$, (ii) enables sufficient plasma confinement for the production of highly charged ions through electron impact ionization, and (iii) suppresses magnetohydrodynamic (MHD) instabilities ${ }^{1}$ by satisfying the conditions $\partial B / \partial r>0$ and $\beta$ $\ll 1$ and where $\beta$ is the ratio of particle pressure $n_{e} k T_{e}$ and magnetic pressure $B^{2} / 2 \mu_{0}$.

The effect of the field strength on the performance of ECR ion sources has been studied extensively. The results have been summarized ${ }^{2}$ in the form of semiempirical scaling laws: $B_{\text {inj }} / B_{\mathrm{ECR}}=4, B_{\mathrm{rad}} / B_{\mathrm{ECR}}=2, B_{\min } \approx 0.4 B_{\mathrm{rad}}$, and $B_{\text {ext }} \approx 0.9 B_{\text {rad }}$, where $B_{\text {inj }}$ and $B_{\text {ext }}$ are the field maxima at injection and extraction, $B_{\text {rad }}$ is the maximum radial field on the magnetic pole, $B_{\min }$ the minimum field value in the plasma chamber, and $B_{\mathrm{ECR}}$ the resonance field for non-relativistic $(\gamma=1)$ electrons. Due to the superposition of the solenoid and sextupole fields, the radial field depends on the solenoid field strength and is not constant in axial direction along the pole. Thus, it is customary to eliminate $B_{\text {rad }}$ from the scaling

Note: Invited paper, published as part of the Proceedings of the 16th International Conference on Ion Sources, New York, New York, USA, August 2015 .

a) olli.tarvainen@jyu.fi laws by writing $B_{\min } / B_{\mathrm{ECR}} \approx 0.8$, which is a well-defined ratio characterizing the magnetic field strength.

Figure 1 shows the performances of VENUS, RAMSES (RIKEN liquid-He-free SC-ECR), SuSI (NSCL), and JYFL $14 \mathrm{GHz}$ A-ECR, operating at frequencies of 14$18 \mathrm{GHz}$, as a function of $B_{\min } / B_{\mathrm{ECR}}$. The given data have been chosen for display because the original references ${ }^{3-5}$ explicitly show the effect of varying $B_{\min } / B_{\mathrm{ECR}}$-ratio on the extracted beam currents. The extracted currents of highly charged ions have been normalized with respect to the maximum current reported for each data set in order to allow the comparison between different ion sources and ion species.

The experimental data in Fig. 1 demonstrate that the best performance is achieved at $0.7 \leq B_{\min } / B_{\mathrm{ECR}} \leq 0.8$, which corresponds approximately the value derived from the semiempirical scaling. ${ }^{2}$ The drop of the performance at higher $B_{\min } / B_{\mathrm{ECR}}$-ratio is accompanied by the appearance of periodic beam current fluctuations at least in the case of SuSI and JYFL $14 \mathrm{GHz}$ A-ECR. It has been shown ${ }^{4}$ that the optimum $B_{\min } / B_{\mathrm{ECR}}$-ratio is independent on the extraction mirror field $B_{\text {ext }}$, for example, which indicates that the decrease of the source performance at $B_{\min } / B_{\mathrm{ECR}} \geq 0.8$ is not due to changing plasma confinement. Furthermore, it has been reported $^{6}$ that the optimum $B_{\min } / B_{\mathrm{ECR}}$-ratio decreases slightly with increasing microwave power, which indicates that its actual value rather depends on electron heating properties. Thus, it is appropriate to ask the question: What limits the ECRIS performance at $B_{\min } / B_{\mathrm{ECR}} \geq 0.8$ ? A possible explanation, consistent with the aforementioned experimental observations and supported by new data, is given in Secs. II-IV. 


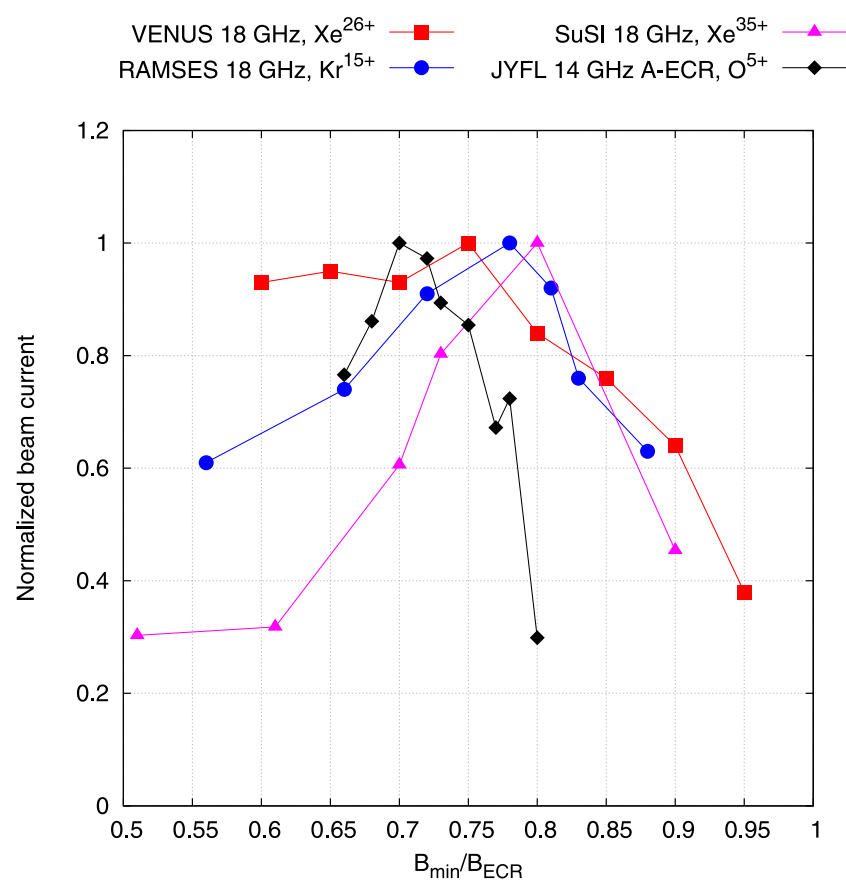

FIG. 1. The performance of VENUS $(18 \mathrm{GHz})$ —, RAMSES $(18 \mathrm{GHz}) \bullet$, SuSI (18 GHz) \ (previously unpublished), and JYFL $14 \mathrm{GHz}$ A-ECR $\bullet$ ion sources $^{3-5}$ as a function of $\boldsymbol{B}_{\min } / \boldsymbol{B}_{\mathrm{ECR}}$-ratio. Lines connecting the data points have been added to guide the eye.

\section{ELECTRON CYCLOTRON INSTABILITIES IN ECR-HEATED PLASMAS}

The electron energy distribution (EED) in ECRIS plasmas is considered to consist of cold, warm, and hot electron populations with $\left\langle E_{e, \text { cold }}\right\rangle=10-100 \mathrm{eV},\left\langle E_{e, \text { warm }}\right\rangle=1-10 \mathrm{keV}$, and $\left\langle E_{e, h o t}\right\rangle \geq 10 \mathrm{keV}^{7,8}$ The warm electron population is responsible for electron impact ionization of highly charged ions and together with the "run-away" hot electron component carries most of the plasma energy content. ${ }^{9}$ Only the perpendicular energy of the electrons increases in the resonance and, hence, the electron velocity distribution (EVD) is strongly anisotropic, i.e., $\left\langle v_{\perp}\right\rangle \gg\left\langle v_{\|}\right\rangle$, where the symbols $\perp$ and $\|$ refer to the external magnetic field. Such EED is prone to kinetic electron cyclotron instabilities. ${ }^{10}$

Kinetic plasma instabilities driven by the anisotropy of the EED have been detected and studied in ECR-heated mirror plasmas $^{11,12}$ and recently in a minimum-B ECRIS. ${ }^{5,13}$ Each onset of the instability is associated with strong microwave emission from the plasma followed by a burst of electrons ejected into the loss cone and thus escaping the magnetic trap. In a magnetic mirror machine, the instabilities are responsible for plasma (energy) losses up to $25 \%,{ }^{9}$ which in an ECRIS would be detrimental for high charge state production. This is because (i) the ion confinement time is reduced, which prohibits the formation of highly charged ions and (ii) the reduced density of warm electrons suppresses the ionization rate.

\section{EXPERIMENTAL SETUP AND RESULTS}

The experimental data demonstrating that the instabilities limit the tuning range available for the optimization of the extracted currents of high charge state ions in cw operation were taken with the A-ECR type JYFL $14 \mathrm{GHz}$ ECRIS $^{14}$ equipped with two solenoid coils, permanent magnet sextupole, and aluminum plasma chamber. The magnetic field strength can be adjusted by varying the solenoid coil currents, which affects the injection and extraction mirror ratios as well as the B-minimum. In Sec. III, the $B_{\min } / B_{\mathrm{ECR}}$-ratio is used for characterizing the magnetic field. The given range of $0.66 \leq B_{\min } / B_{\mathrm{ECR}} \leq 0.8$ corresponds to injection and extraction fields ranging from $1.98 \mathrm{~T} \leq B_{\text {inj }} \leq 2.13 \mathrm{~T}$ and $0.9 \mathrm{~T}$ $\leq B_{\text {ext }} \leq 1.02 \mathrm{~T}$, respectively. The biased disc voltage was kept at $80-150 \mathrm{~V}$ (negative).

The following diagnostics tools were used for detecting the instabilities and characterizing their effect on the extracted beam currents. A thorough description of the experimental setup is presented elsewhere. ${ }^{5,13}$

- Microwave detector diode (0.01-50 GHz, $10 \mathrm{~ns}$ resolution) connected to a WR75 waveguide port at the injection of the ion source.

- Bismuth germanate (BGO) scintillator coupled with a current-mode photomultiplier tube (PMT) (Na doped CsI). The scintillator is placed outside the plasma chamber near a radial diagnostics port.

- A Faraday cup $\sim 5 \mathrm{~m}$ downstream in the beamline.

These complimentary diagnostics yield information on the hot electrons interacting with the plasma electromagnetic (EM)-wave and emitting microwave radiation, wall bremsstrahlung (power) flux induced by energetic electrons escaping the confinement, and extracted, $m / q$-analyzed beam currents. Figure 2 shows representative examples of the diagnostics signals recorded with an oxygen plasma.

The sequence of observable events starts at $t=0$ by strong microwave emission from the plasma lasting some hundreds of ns (Fig. 2(a)). The microwave emission is caused by the hot electrons interacting resonantly with the excited plasma wave. It has been observed that the emission frequencies are almost independent of the plasma parameters and are always lower than $14 \mathrm{GHz}$ (dominant frequencies are 12.52 and $11.09 \mathrm{GHz}$ ), which is the frequency used for electron heating. ${ }^{15}$ These observations imply that the excited plasma wave mode is slow extraordinary Z-mode propagating quasi-longitudinally with respect to the external magnetic field. ${ }^{15}$ The microwave emission coincides with a burst of (wall) bremsstrahlung exceeding the continuous bremsstrahlung flux by an order of magnitude at least. The bremsstrahlung peak (Fig. 2(b)) lasting some tens of $\mu \mathrm{s}$ is caused by hot electrons, expelled from the magnetic confinement due to the interaction with the plasma wave. It is of note that such burst of bremsstrahlung saturates even the fastest energy-resolving detectors (e.g., germanium) with a typical signal processing times of 1-2 $\mu$ s. Therefore, it is impossible to measure the bremsstrahlung energy spectrum during the instability (the scintillator + PMT detector measures power flux). While the microwave emission and burst of energetic electrons are direct signatures of the electron cyclotron instability, the perturbation of the extracted ion currents is an inevitable outcome. The extracted currents of highly charged ions (e.g., $\mathrm{O}^{6+}$ shown in Fig. 2(c)) decrease abruptly 

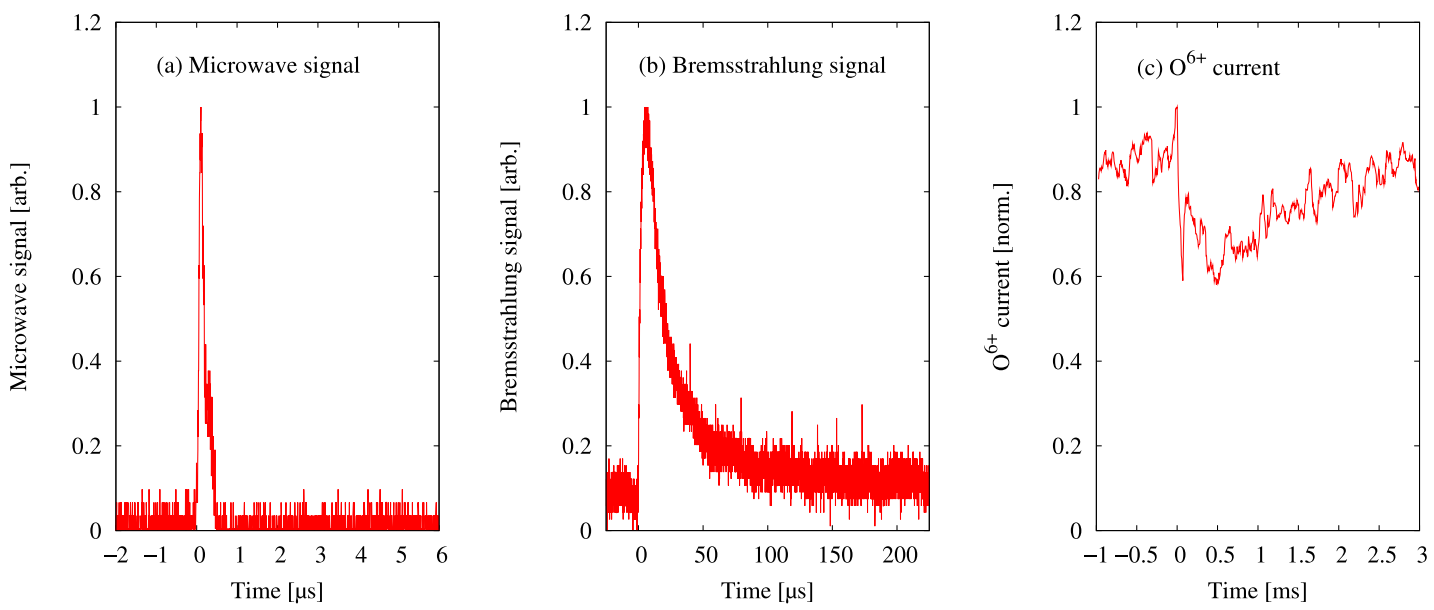

FIG. 2. Examples of (a) microwave emission, (b) bremsstrahlung and (c) normalized $\mathrm{O}^{6+}$ beam current signals. Instability onset occurs at $t=0$. Note different time scales in each subfigure.

following the instability and then enter a slower recovery phase.

The instabilities expel a significant fraction of the hot and warm electrons, ${ }^{9}$ which results to drastic increase of the plasma potential balancing the electron and ion losses. This claim is supported by the measurement of the ion beam energy spread, $\Delta E / E$, during the instability-induced transient. Figure 3 shows the temporally resolved energy spread of $\mathrm{Ar}^{9+}$ beam with $t$ $=0$ corresponding to the leading edge of the microwave and bremsstrahlung burst. The data were obtained by sweeping the $90^{\circ} \mathrm{m} / \mathrm{q}$-analyzing magnet and recording the corresponding beam current signals with the Faraday cup at $10 \mathrm{kV}$ extraction voltage. Immediately following the instability, the $\mathrm{Ar}^{9+}$ peak of the $m / q$-spectrum stretches in energy and overlaps with the adjacent $\mathrm{Ar}^{8+}$ peak for some $\mu \mathrm{s}$. This corresponds to $\Delta E / E$ of $12 \%$ as a minimum and implies that the plasma potential reaches values $\geq 1 \mathrm{kV}$ during the transient. The tail on the left-hand side is due to $\mathrm{Ar}^{10+}$. Similar transient values were recorded with oxygen and helium. Moreover, the level of impurities, e.g., carbon, in the $m / q$-spectrum increases after each instability event, which can be explained by adsorption (sputtering) from the plasma chamber walls by energetic ions repelled by the plasma potential.

So far, we have concentrated on a single instability event and associated diagnostics signals in 0.1-100 $\mu$ s timescale.

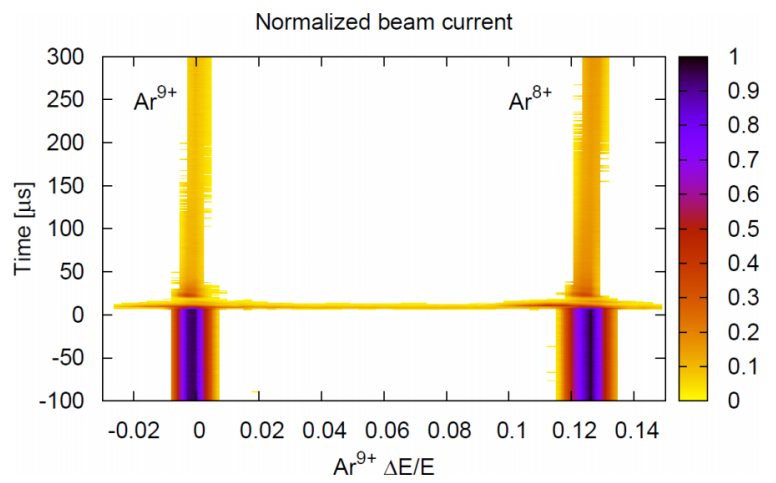

FIG. 3. The energy spread of $\mathrm{Ar}^{9+}$ ion beam. The instability onset occurs at $t=0$. The horizontal axis represents the magnetic field sweep of the $m / q$-analyzing magnet.
However, ion beams extracted from ECRISs often suffer from periodic ripple at ms-scale, i.e., at repetition rate on the order of $10^{2}-10^{3} \mathrm{~Hz} .{ }^{16}$ It has been demonstrated that such oscillations can be explained by periodic onsets of the cyclotron instabilities. ${ }^{5}$ From the ECRIS performance point-of-view, the most relevant question is what affects the transition from stable to unstable operating regime and the repetition rate of the periodic instabilities?

The instabilities are triggered when their growth rate, which is proportional to the degree of the anisotropy of the EEDF, exceeds their damping rate, which is proportional to electron collision frequency. The most critical tuning parameter affecting the appearance of the cyclotron instabilities is the (solenoid) magnetic field strength. The exact threshold magnetic field depends also on the gas species, microwave power, neutral gas pressure, and source potential. ${ }^{17}$ The effect of the magnetic field strength is demonstrated in Fig. 4 showing the instability threshold $B_{\min } / B_{\mathrm{ECR}}$ as a function of microwave power for different noble gases ( $\mathrm{He}, \mathrm{Ar}$, and $\mathrm{Xe}$ ). The data were taken by adjusting the total extracted currents of different plasma species to be $1.2 \mathrm{~mA}$ at $10 \mathrm{kV}$ source potential suggesting that the plasma densities are approximately the same in each case. The error bars represent the step used for adjusting the magnetic field. It is believed that the plasma species

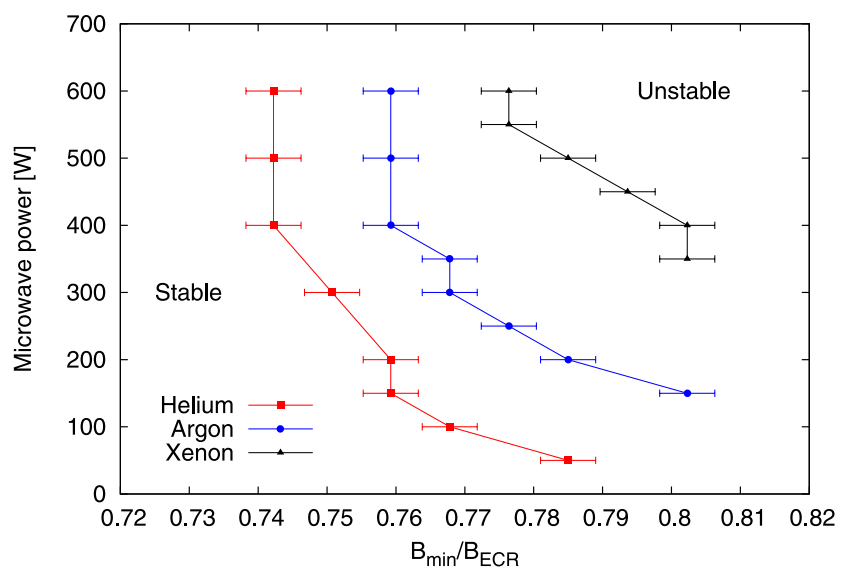

FIG. 4. The instability threshold $B_{\min } / B_{\mathrm{ECR}}$ of helium, argon, and xenon plasmas as a function of microwave power. 

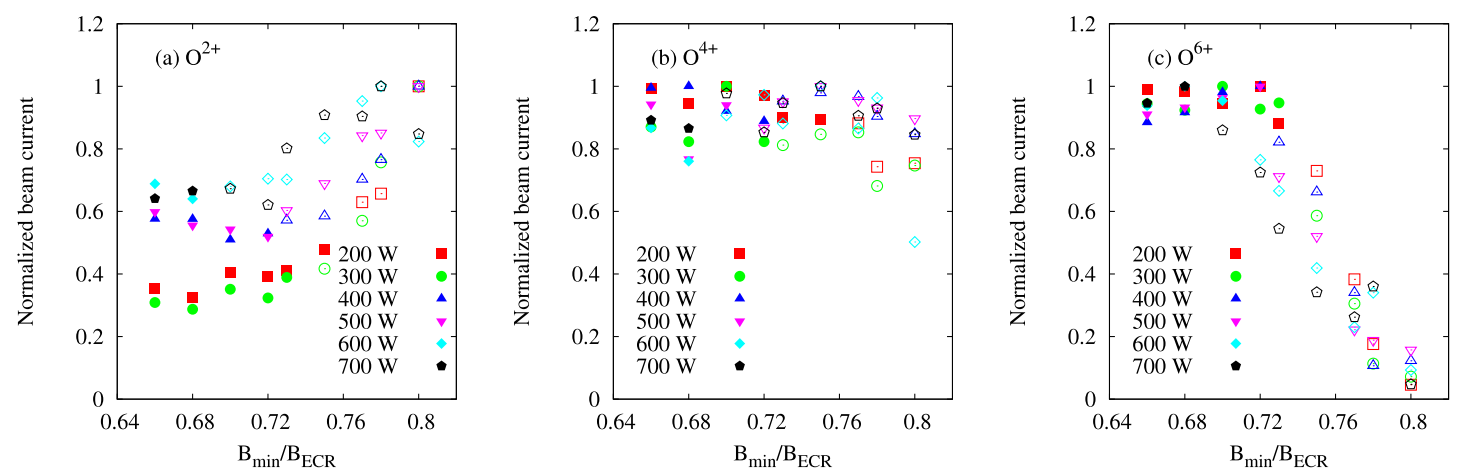

FIG. 5. Normalized currents of (a) $\mathrm{O}^{2+}$, (b) $\mathrm{O}^{4+}$, and (c) $\mathrm{O}^{6+}$ ion beams as a function of $B_{\min } / B_{\mathrm{ECR}}$. Solid symbols correspond to stable and open symbols to unstable operating regime. The data are collected at $4.9 \times 10^{-7}$ mbars neutral gas pressure.

dependence is related to increased rate of inelastic collisions in plasmas consisting of ions with high atomic number.

The extracted currents of highly charged ions are limited by the instabilities above the threshold $B_{\min } / B_{\mathrm{ECR}}$-ratio. This is demonstrated in Fig. 5 showing the normalized, temporally averaged currents (over several seconds) of $\mathrm{O}^{2+}, \mathrm{O}^{4+}$, and $\mathrm{O}^{6+}$ ion beams as a function of the $B_{\min } / B_{\mathrm{ECR}}$-ratio at different microwave powers typical for the JYFL $14 \mathrm{GHz}$ ECRIS operation. The data sets chosen for display represent a small fraction of the data, ${ }^{5}$ collected at various microwave powers and neutral gas pressures for several charge states of different elements, supporting the conclusions made hereafter. The normalization is carried out for each data set in order to eliminate the effect of microwave power, i.e., the increase of extracted currents of highly charged ions with increasing power, from the presented data. This highlights the fact that the occurrence of electron cyclotron instabilities above the threshold B-field restricts the parameter space available for the optimization of high charge state ion currents and is likely to explain the trend in Fig. 1.

The extracted currents of the high charge states, i.e., $\geq \mathrm{O}^{4+}$, decrease drastically with increasing $B_{\min } / B_{\mathrm{ECR}}$-ratio above the instability threshold. On the contrary, the extracted currents of low charge states, such as $\mathrm{O}^{2+}$, are higher in the unstable operating regime, which indicates that the charge state distribution of the plasma shifts substantially due to the periodic perturbations. The transition to the unstable regime occurs at $0.7 \leq B_{\min } / B_{\mathrm{ECR}} \leq 0.75$. In the unstable regime, the source performance cannot be restored by varying the injected power or neutral gas pressure. The charge state dependence can be explained by the fact that the temporal interval between periodic instabilities is shorter than the production and confinement times of highly charged ions. The repetition rate of the instabilities varies on the order of $10^{2}-10^{3} \mathrm{~Hz}$, which corresponds to temporal period of 1-10 ms. The production times of highly charged ions in ECRIS plasmas are several tens of $\mathrm{ms}^{18}$ while their confinement times are typically several ms. ${ }^{8}$ This means that in order to reach high charge states the ions must survive numerous instability events without being expelled from the confinement following the burst of electrons. The repetition rate of the instabilities increases with increasing magnetic field strength (or $B_{\min } / B_{\mathrm{ECR}}$-ratio), which reduces the probability for highly charged ion production and affects the charge state distribution of extracted ion beams above the instability threshold as shown in Fig. 5.

\section{DISCUSSION}

It has been demonstrated that kinetic instabilities limit the performance of the JYFL $14 \mathrm{GHz}$ ECRIS in terms of intensity and stability of the extracted beam current. It is worth noting that several ECRISs (e.g., VENUS and NIRS-HEC) have been reported to suffer from periodic oscillations of extracted beam current, ${ }^{16,19}$ which are most likely due to electron cyclotron instabilities.

The appearance of the instabilities depends strongly on the anisotropy of the EVD. The electron heating in ECRIS plasmas is a stochastic process. The stochastic and adiabatic limits ${ }^{20}$ of the electron energy at a given microwave frequency depend on the distribution of the magnetic field gradient, $\nabla B_{\mathrm{ECR}}$ (more precisely the parallel component of the gradient with respect to the external magnetic field, $\left.\frac{\vec{B}}{|\vec{B}|} \cdot \nabla \vec{B}\right)$, and strength of the electric field, $E_{\mathrm{ECR}}$, at the resonance. The effect of the magnetic field gradient on the electron energies and anisotropy of the EVD is stronger than the effect of the electric field, especially if the parallel resonance gradient component, $\frac{\vec{B}}{|\vec{B}|} \cdot \nabla \vec{B}$, is close to zero. ${ }^{21}$ In a minimum-B configuration realized with two solenoids (e.g., the JYFL $14 \mathrm{GHz}$ ECRIS), the distribution of this component shifts drastically toward lower values with increasing $B_{\min } / B_{\mathrm{ECR}} .{ }^{5}$ On the other hand, the electric field strength at the resonance depends on the applied microwave power. Hence, the observed trends, namely, the shift of the instability threshold with $B_{\mathrm{min}} / B_{\mathrm{ECR}}$ and microwave power, can be explained by the resonant nature of the electron heating.

The minimum-B configuration based on the superposition of solenoid and sextupole fields is effective in suppressing MHD-instabilities but has the inherent drawback of excessive electron heating. For stable operation of an ECRIS, it is important to avoid having too low gradient at the resonance surface. Therefore, it is possible that the appearance of the instabilities could be prevented by minimizing the zero-gradient regions on the resonance surface by a careful design of the magnetic field. Another possible method to extend the parameter space of the stable operating regime is to apply multiple frequency heating. It has been shown that the oscillations of the extracted beam current can be mitigated by two-frequency heating. ${ }^{19}$ The effect has been recently attributed to suppression of the electron cyclotron instabilities ${ }^{22}$ due to interaction between the secondary microwave radiation and the hot electron component of the ECRIS plasma. 


\section{ACKNOWLEDGMENTS}

This work has been supported by the EU 7th framework program Integrating Activities - Transnational Access, Project No. 262010 (ENSAR), the Academy of Finland under the Finnish Centre of Excellence Program 2012-2017 (Nuclear and Accelerator Based Physics Research at JYFL), and researcher mobility Grant Nos. 285999 and 285895.

${ }^{1}$ T. Antaya and S. Gammino, Rev. Sci. Instrum. 65(5), 1723 (1994).

${ }^{2}$ D. Hitz, A. Girard, G. Melin, S. Gammino, G. Ciavola, and L. Celona, Rev. Sci. Instrum. 73, 509 (2002).

${ }^{3}$ D. Leitner, C. M. Lyneis, T. Loew, D. S. Todd, S. Virostek, and O. Tarvainen, Rev. Sci. Instrum. 77(3), 03A302 (2006).

${ }^{4}$ H. Arai, M. Imanaka, S. M. Lee, Y. Higurashi, T. Nakagawa, M. Kidera, T. Kageyama, M. Kase, Y. Yano, and T. Aihara, Nucl. Instrum. Methods Phys. Res., Sect. A 491(1-2), 9 (2002).

${ }^{5}$ O. Tarvainen, J. Laulainen, J. Komppula, R. Kronholm, T. Kalvas, H. Koivisto, I. Izotov, D. Mansfeld, and V. Skalyga, Rev. Sci. Instrum. 86, 023301 (2015)

${ }^{6}$ T. Nakagawa, M. Kidera, Y. Higurashi, J. Ohnishi, T. Kageyama, T. Aihara, A. Goto, and Y. Yano, High Energy Phys. Nucl. Phys. 31(1), 133 (2007).

${ }^{7}$ C. Barue, M. Lamoreux, P. Briand, A. Girard, and G. Melin, J. Appl. Phys. 76, 2662 (1994).

${ }^{8}$ G. Douysset, H. Khodja, A. Girard, and J. P. Briand, Phys. Rev. E 61, 3015 (2000).

${ }^{9}$ S. A. Hokin, R. S. Post, and D. L. Smatlak, Phys. Fluids B 1, 862 (1989).

${ }^{10}$ S. V. Golubev and A. G. Shalashov, Phys. Rev. Lett. 99, 205002 (2007).
${ }^{11}$ R. C. Garner, M. E. Mauel, S. A. Hokin, R. S. Post, and D. L. Smatlak, Phys. Fluids B 2(2), 242 (1990).

${ }^{12}$ M. Viktorov, D. Mansfeld, and S. Golubev, EPL 109, 65002 (2015).

${ }^{13}$ O. Tarvainen, I. Izotov, D. Mansfeld, V. Skalyga, S. Golubev, T. Kalvas, H. Koivisto, J. Komppula, R. Kronholm, J. Laulainen, and V. Toivanen, Plasma Sources Sci. Technol. 23, 025020 (2014).

${ }^{14}$ H. Koivisto, P. Heikkinen, V. Hänninen, A. Lassila, H. Leinonen, V. Nieminen, J. Pakarinen, K. Ranttila, J. Ärje, and E. Liukkonen, Nucl. Instrum. Methods Phys. Res., Sect. B 174, 379 (2001).

${ }^{15}$ I. Izotov, O. Tarvainen, D. Mansfeld, V. Skalyga, H. Koivisto, T. Kalvas, J. Komppula, R. Kronholm, and J. Laulainen, Plasma Sources Sci. Technol. 24, 045017 (2015).

${ }^{16}$ O. Tarvainen, T. Kalvas, H. Koivisto, J. Komppula, V. Toivanen, C. M. Lyneis, and M. M. Strohmeier, in Proceedings of the 20th International Workshop on ECRIS, Sydney, Australia, 2012, TUXO02, http://accelconf. web.cern.ch/AccelConf/ECRIS2012/papers/tuxo02.pdf.

${ }^{17} \mathrm{O}$. Tarvainen et al., in Proceedings 21st International Workshop on ECRIS, Nizhny Novgorod, Russia, 2014, MOOBMH01 http://accelconf.web.cern. ch/AccelConf/ECRIS2014/papers/moobmh01.pdf.

${ }^{18}$ R. C. Vondrasek, R. H. Scott, R. C. Pardo, and D. Edgell, Rev. Sci. Instrum. 73, 548 (2002).

${ }^{19}$ A. Kitagawa, T. Fujita, M. Muramatsu, S. Biri, R. Racz, Y. Kato, K. Yano, N. Sasaki, and W. Takasugi, in Proceedings of the 20th International Workshop on ECRIS, Sydney, Australia, 2012, TUXO03, http://accelconf. web.cern.ch/AccelConf/ECRIS2012/papers/tuxo03.pdf.

${ }^{20}$ N. C. Wyeth, A. J. Lichtenberg, and M. A. Lieberman, Plasma Phys. 17, 679 (1975).

${ }^{21}$ M. C. Williamson, A. J. Lichtenberg, and M. A. Lieberman, J. Appl. Phys. 72, 3924 (1992).

${ }^{22}$ V. Skalyga, I. Izotov, T. Kalvas, H. Koivisto, J. Komppula, R. Kronholm, J. Laulainen, D. Mansfeld, and O. Tarvainen, Phys. Plasmas 22, 083509 (2015). 